\title{
A Poly(ethylene glycol) Gradient for Biochip Development
}

\author{
Andréas Larsson and Bo Liedberg* \\ Division of Sensor Science and Molecular Physics, Department of Physics, Chemistry and Biology, \\ Linköping University, SE-581 83 Linköping, Sweden
}

Supporting Information 
The supporting figures herein contain IR RA spectra along gradients of a PEG-based matrix, which was UV graft polymerized on different platforms. The data was acquired using a Bruker Hyperion 3000 microscope equipped with a grazing angle objective and a high-precision motorized sample table.

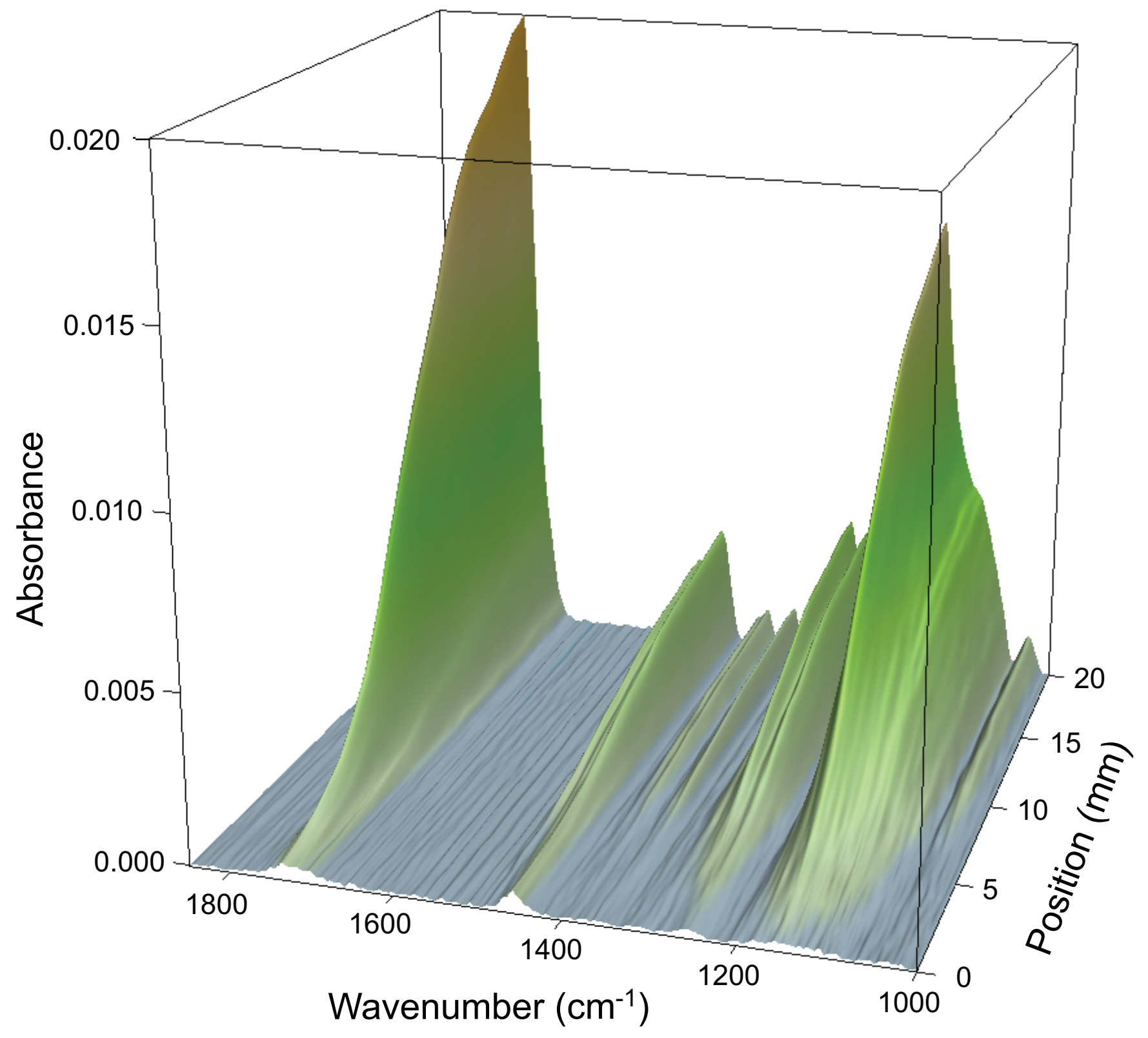

Supporting Figure 1. Spatially resolved 3D plot of IR RA spectra from measurements on a nonmodified, $20 \mathrm{~mm}$ long matrix gradient UV graft polymerized onto a COP platform. 


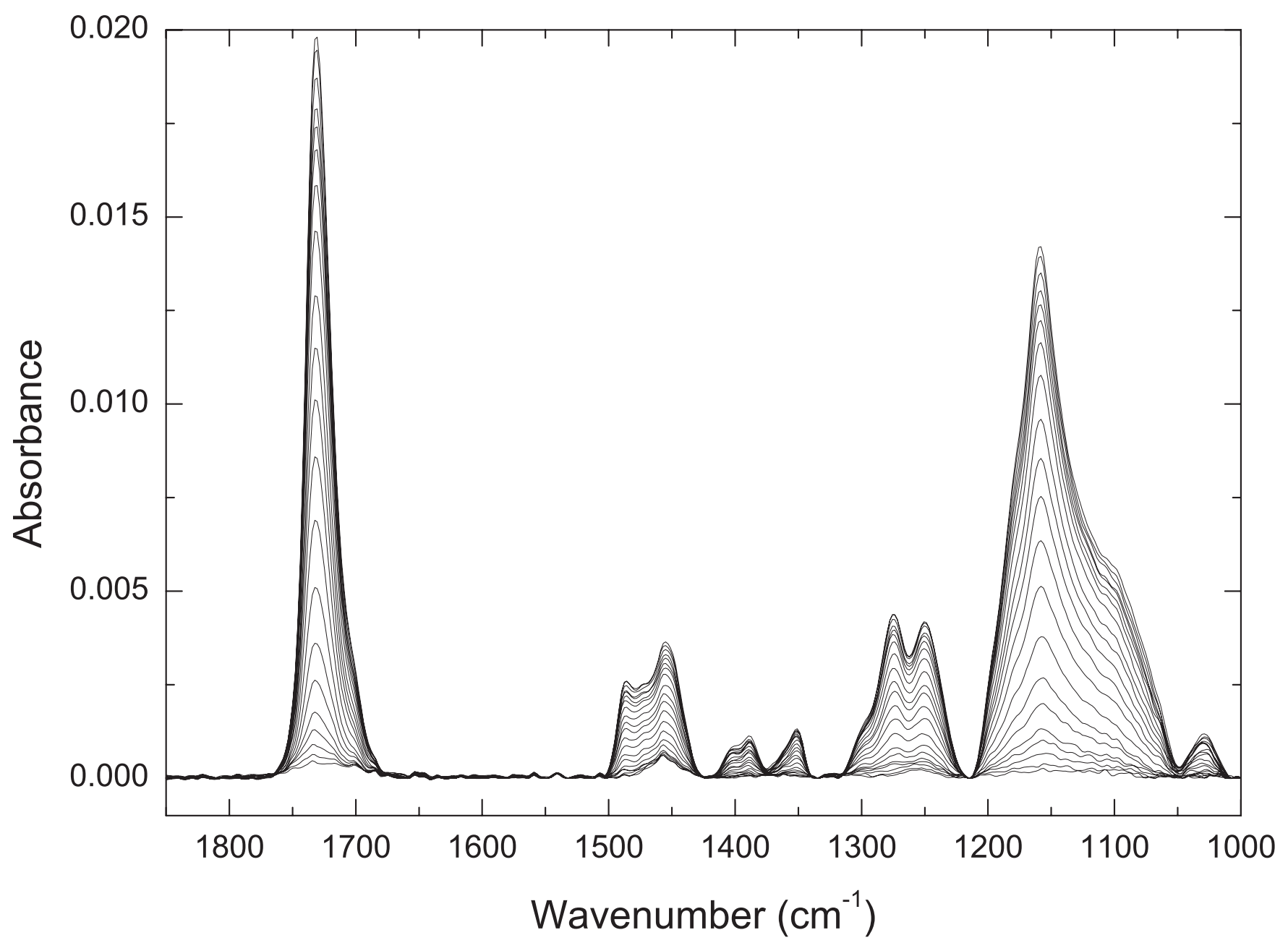

Supporting Figure 2. Infrared RA spectra from measurements on a non-modified, $20 \mathrm{~mm}$ long matrix gradient UV graft polymerized onto a COP platform. 


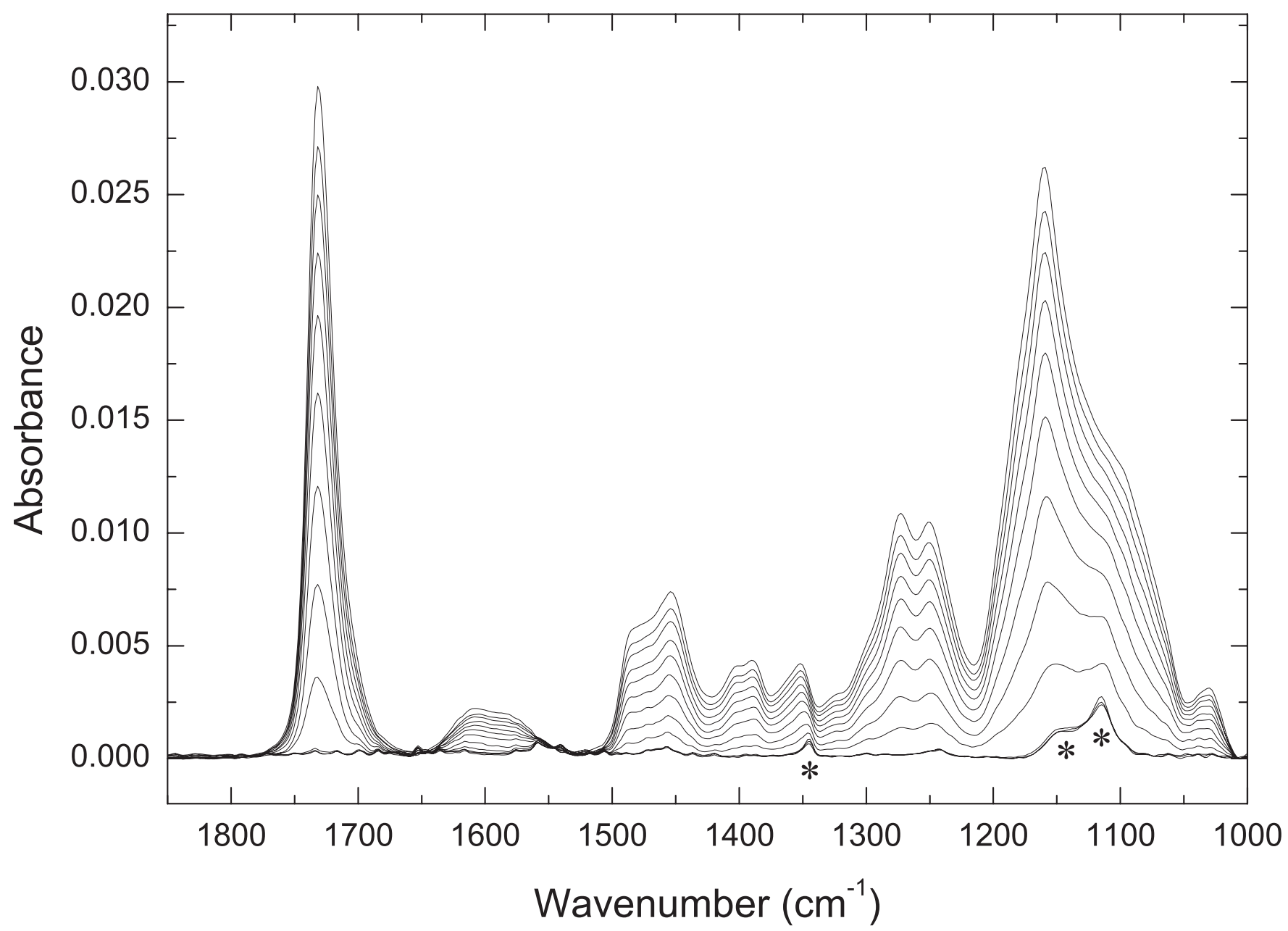

Supporting Figure 3. Infrared RA spectra from measurements on a carboxylated, $7 \mathrm{~mm}$ long matrix gradient UV graft polymerized onto a thiol SAM platform. The peaks marked with '*' correspond to helical $\left(1345\right.$ and $\left.1115 \mathrm{~cm}^{-1}\right)$ and all trans $\left(1146 \mathrm{~cm}^{-1}\right)$ conformers ${ }^{1}$ of the oligo EG segments in the supporting HS-UDA-mPEG 10 monolayer. 


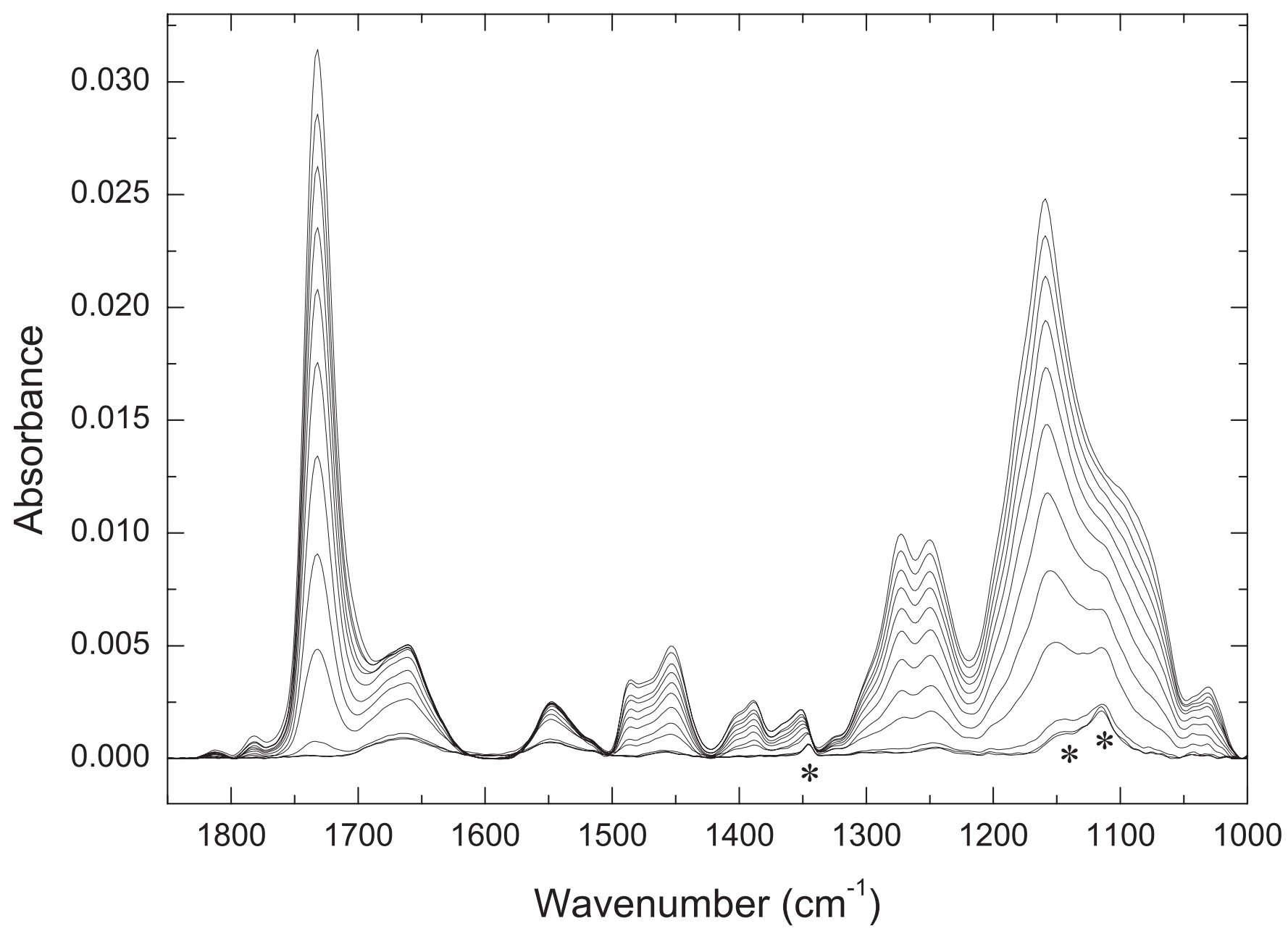

Supporting Figure 4. Infrared RA spectra from measurements on a $7 \mathrm{~mm}$ long matrix gradient onto which HFib was immobilized. The UV graft polymerization platform was a thiol SAM. The peaks marked with ' $*$ ' correspond to helical $\left(1345\right.$ and $\left.1115 \mathrm{~cm}^{-1}\right)$ and all trans $\left(1146 \mathrm{~cm}^{-1}\right)$ conformers ${ }^{1}$ of the oligo EG segments in the supporting HS-UDA-mPEG ${ }_{10}$ monolayer. 


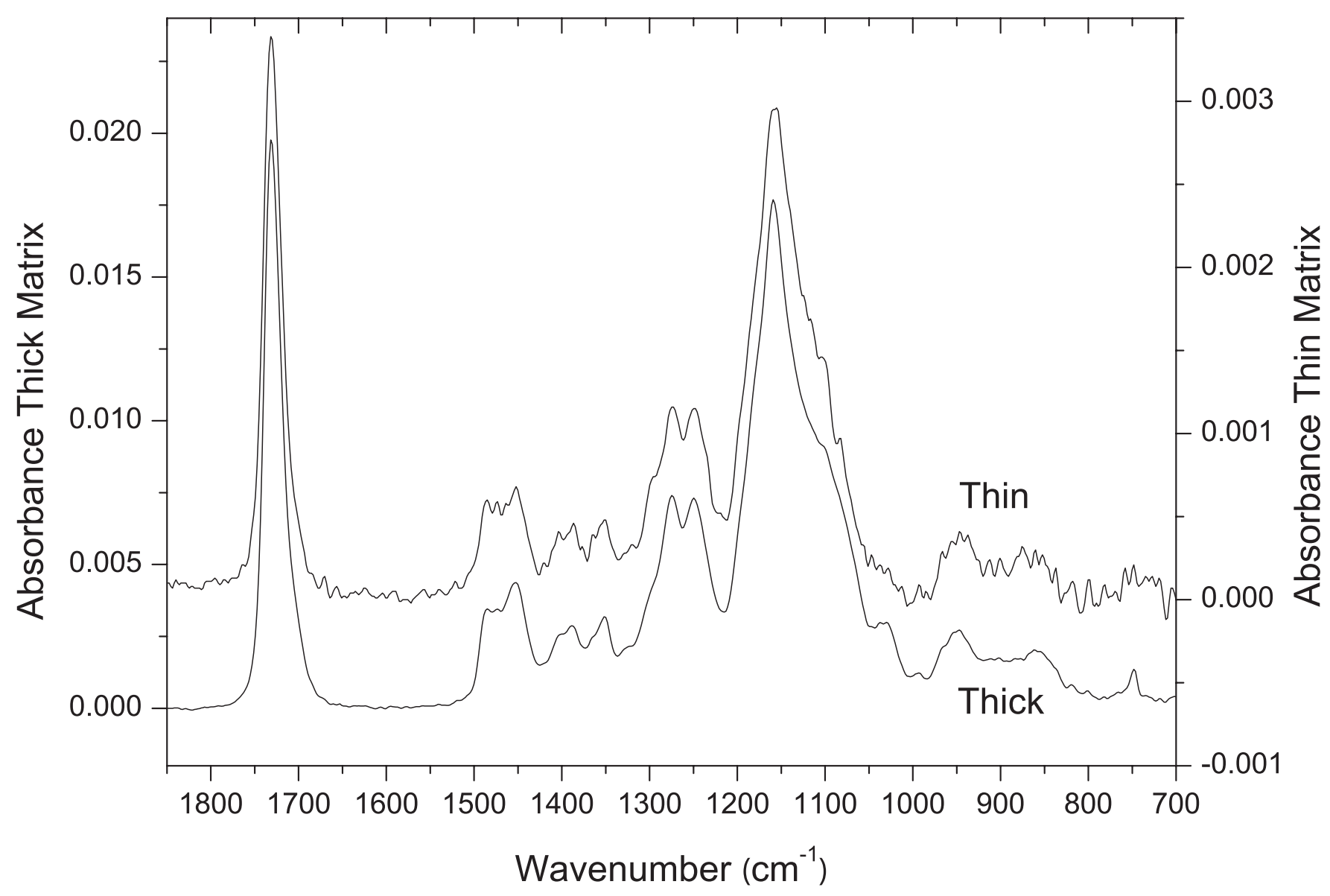

Supporting Figure 5. Comparison between infrared RA spectra from measurements on thin and thick parts of a matrix gradient, graft polymerized onto a COP platform. The "thin" spectrum is indistinguishable (except for noise) from the "thick" spectrum (the "thin" and "thick" spectra have different scales for better comparison).

\section{Reference}

(1) Valiokas, R.; Svedhem, S.; Svensson, S. C. T.; Liedberg, B. Langmuir 1999, 15, 3390-3394. 